\title{
A SIMPLE METHOD FOR DETERMINING OPTIMUM CORRECTIONS FOR HIGH-ACCURACY EPMA IN DIFFICULT CHEMICAL SYSTEMS
}

\author{
J. T. Armstrong ${ }^{*}$, R. B. Marinenko ${ }^{*}$, and J. M. Davis *** \\ *Surface and Microanalysis Science Division, National Institute of Standards and Technology, \\ Gaithersburg, MD 20899-8371 \\ *** Undergraduate School of Engineering, Clemson University, Clemson, SC 296340911
}

Increasingly, analysts are called to perform high accuracy quantitative electron microprobe analyses using X-ray lines for which the available correction procedures have significant uncertainties. Conventional evaluation of microprobe corrections typically involves analyzing a series of standards as 'unknowns' (often at several different beam energies), correcting the data through the algorithms and parameters being tested, and plotting the results as error histograms. The problem with this method is that often there are simply not enough multielement, homogeneous materials available to provide the necessary suite of secondary standards. Even ifthere are, independent determination of the exact compositions and degree of homogeneity of the candidate standards requires so much time, effort, and money as to make it impractical.

We propose a simplified version of the multi-keV evaluation procedure for binary systems that does not require a series of homogeneous secondary standards. In a specimen $\mathrm{A}_{\mathrm{x}} \mathrm{B}_{1-\mathrm{x}}$ (where $\mathrm{A}$ and $\mathrm{B}$ are single elements or compounds of fixed atom proportions), as long as $A+B=100 \%--$ a criterion easily checked by qualitative analysis, there should be a simple monotonic relation between the measured k-factor (relative intensity of the specimen to an end-member standard) and the relative concentration at any single set of analytical conditions. If one measures kvalues at two different electron beam energies for a series of samples having different $\mathrm{A} / \mathrm{B}$ concentration ratios (or a series different points on a inhomogeneous specimen of $\mathrm{A}: \mathrm{B}$ ), one should obtain monotonic curves (plotting the k-values of $\mathrm{A}$ or $\mathrm{B}$ at one $\mathrm{E}_{0}$ vs. those at the other $\mathrm{E}_{0}$ ) having end points of 0 and 1 (e.g., Fig. 1). The degree of curvature of the hyperbolic-like curve stretching from 0 to 1 (or the slope of a near-linear portion of the curve over a limited range of concentration) will depend on the difference in the magnitude of the correction factors for the two sets of analytical conditions. Bad analyses should appear as points lying off the curve formed by the majority of the data 2 .

This $\mathrm{k}\left(\mathrm{E}_{0,1}\right)$ vs $\mathrm{k}\left(\mathrm{E}_{0,2}\right)$ plot can be used to evaluate the agreement to a series of correction algorithms without knowing, a priori, the compositions of any of the individual points. One simply calculates the k-values for a range of compositions in the binary system at the two beam energies for each of the correction procedures being considered (e.g., Fig. 2) and plots them along with the experimentally measured points. The expressions can be individually tested for each xray line analyzed and for each pair of electron beam energies employed. If a particular correction procedure well fits the analytical data, one can make the initial assumption that it would be the correction method that would most accurately correct the experimental data (e.g., Fig. 3). One can then process the measured data at the two beam energies using this 'best fit' method and evaluate the quality of the results on the basis of any independent knowledge of the samples being analyzed. The only absolute requirements of this method are that the sample is homogeneous over large enough areas and that the instrument is stable enough so that the microvolumes analyzed are of the same composition for the given point measured at the two different beam energies. 
We are using this method to evaluate a series of materials involved in standard reference material and interactive reference material development at NIST, including the $\mathrm{Cu}_{\mathrm{x}} \mathrm{Au}_{1-\mathrm{x}}, \mathrm{Si}_{\mathrm{x}} \mathrm{Ge}_{1-\mathrm{x}}, \mathrm{Ni}_{\mathrm{x}} \mathrm{Al}_{1-\mathrm{x}}$, and $\mathrm{Al}_{\mathrm{x}} \mathrm{Ga}_{1-\mathrm{x}} \mathrm{As}$ systems.

\section{References}

1. J.T. Armstrong, in Electron Probe Quantitation, K.F.J. Heinrich and D.E. Newbury, Eds., New York, Plenum Press (1991) 261; G.F. Bastin and H.J.M. Heijligers, ibid., 145; J-L. Pouchou and F. Pichoir, ibid., 31.

2. J.T. Armstrong, Microscopy and Microanalysis $\underline{7}$ (suppl. 2) (2001), 670.
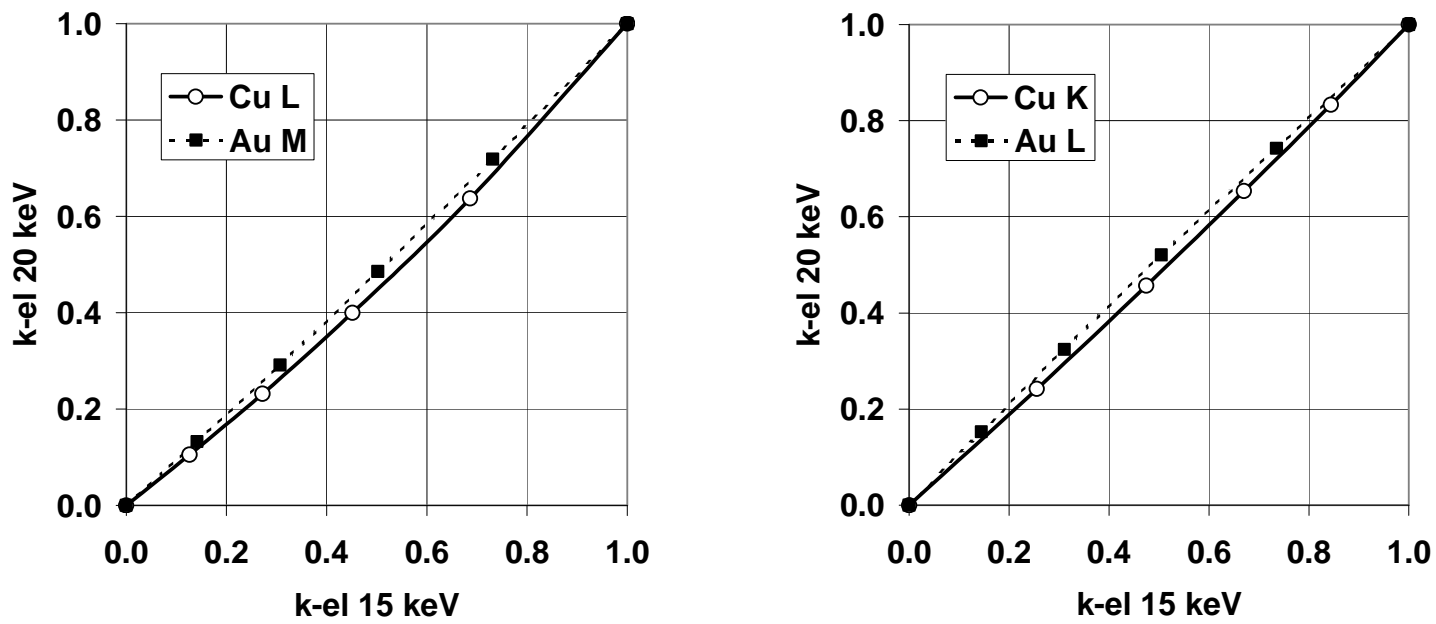

FIG. 1. Measured k-ratios (relative to the pure elements) for the $\mathrm{Cu} \mathrm{L} \mathrm{Cu} \mathrm{K,} \mathrm{Au} \mathrm{L}$ and $\mathrm{Au} \mathrm{M} \mathrm{x}$-ray lines in NIST SRM $482(100 \%, 80: 20,60: 40,40: 60,20: 80$, and $0 \mathrm{wt} \% \mathrm{CuAu}$ alloys) at 15 and 20 $\mathrm{keV}$. The measurement uncertainties are smaller than the size of the plotting symbols. The deviation of the data from a 1:1 line is indicative of the differences in matrix corrections for the two different beam energies. Note that the concentrations of the samples are not needed for this plot.

Measured AIGaAs k-ratios: Al K

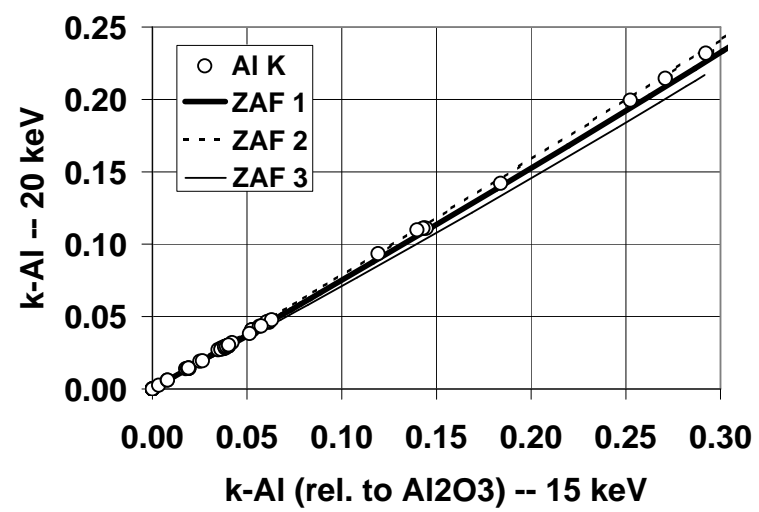

FIG. 2. Measured k-ratios of $\mathrm{Al}$ in a series of
Measured AIGaAs k-ratios: Ga L \& As L

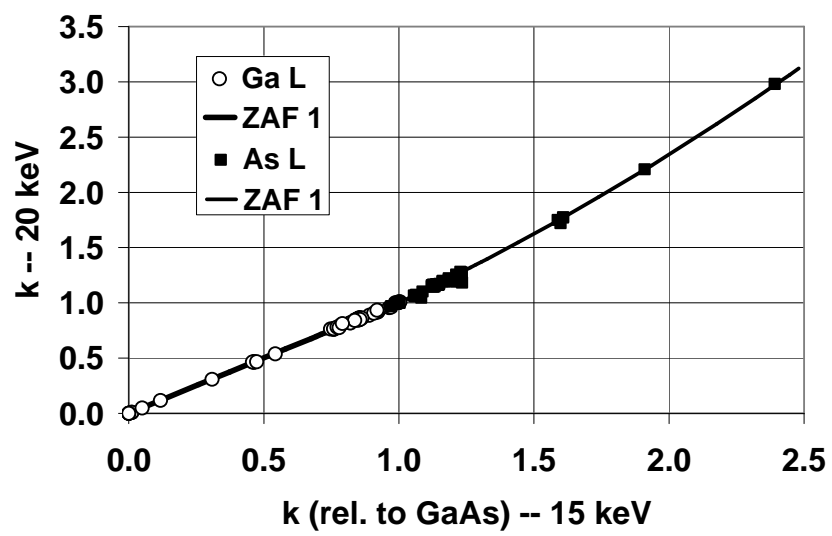

FIG. 3. Measured k-ratios of Ga L and As $\mathrm{L}$ in AlGaAs samples being evaluated for a NIST SRM the same series of samples as shown in Fig. 2 and $\mathrm{k}$-ratios calculated by various commonly used $(\mathrm{k}-\mathrm{Ga}<1, \mathrm{k}-\mathrm{As}>1)$. The same correction correction procedures (whose values range by over procedure, 'ZAF 1', that best fits the measured $20 \%$ relative). 'ZAF 1' fits data to better than $3 \%$. k-Al, also best fits the $\mathrm{Ga} \mathrm{L}$ and $\mathrm{As} \mathrm{L}$ data. 